\title{
The transrectus sheath preperitoneal mesh repair for inguinal hernia: technique, rationale, and results of the first 50 cases
}

\author{
G. G. Koning • C. S. Andeweg • \\ F. Keus • M. W. A. van Tilburg • \\ C. J. H. M. van Laarhoven • W. L. Akkersdijk
}

Received: 29 June 2011 / Accepted: 13 November 2011 / Published online: 1 December 2011

(c) The Author(s) 2011. This article is published with open access at Springerlink.com

\begin{abstract}
Introduction Laparoscopic and endoscopic hernia repair popularized the preperitoneal mesh position due to promising results concerning less chronic pain. However, considerable proportions of severe adverse events, learning curves, or added costs have to be taken into account. Therefore, open preperitoneal mesh techniques may have more advantages. The open approach to the preperitoneal space (PPS) according to transrectus sheath preperitoneal (TREPP) mesh repair is through the sheath of the rectus abdominus muscle. This technique provides an excellent view of the PPS and facilitates elective or acute hernia reduction and mesh positioning under direct vision. In concordance with the promising transinguinal preperitoneal inguinal hernia repair experiences in the literature, we investigated the feasibility of TREPP.

Methods A rationale description of the surgical technique, available level of evidence for thoughts behind technical considerations. Furthermore, a descriptive report of the clinical outcomes of our pilot case series including 50 patients undergoing the TREPP mesh repair.

Results A consecutive group of our first 50 patients were operated with the TREPP technique. No technical problems were experienced during the development of this technique. No conversions to Lichtenstein repair were necessary. No
\end{abstract}

G. G. Koning · F. Keus · C. J. H. M. van Laarhoven

Department of Surgery, Radboud University Nijmegen Medical Centre, Nijmegen, The Netherlands

C. S. Andeweg · M. W. A. van Tilburg · W. L. Akkersdijk (ه) Department of Surgery, St. Jansdal Hospital,

Wethouder Jansenlaan 90, 3844 DG Harderwijk, The Netherlands

e-mail:wl.akkersdijk@stjansdal.nl recurrences and no chronic pain after a mean follow-up of 2 years were notable findings.

Conclusion This description of the technique shows that the TREPP mesh repair might be a promising method because of the complete preperitoneal view, the short learning curve, and the stay-away-from-the-nerves principle. The rationale of the TREPP repair is discussed in detail.

Keywords Inguinal hernia $\cdot$ Preperitoneal $\cdot$ Open · Repair $\cdot$ Mesh $\cdot$ Posterior

\section{Introduction}

After recurrences have been reduced in inguinal hernia repair since the use of mesh, chronic pain is considered to be the most important clinical evaluation after inguinal hernia surgery. Surgery-related factors which may be associated with chronic pain mainly involve nerve injury (or stretching) possibly caused by the surgical approach or the use of mesh fixation devices [1]. Therefore, it may be logical to develop a technique that minimizes or completely avoids nerve contact and does not need mesh fixation. Recently, Reinpold et al. published recommendations for nerve management during surgery [2]. We developed and investigated an easy open preperitoneal technique that may fulfil these recommendations. This open transrectus sheath preperitoneal approach (TREPP) differs essentially from other open preperitoneal techniques, such as the transinguinal preperitoneal (TIPP) technique [3-5]. The TIPP technique is possibly associated with less chronic pain because of its preperitoneal mesh position and may be associated with less adverse events compared to Lichtenstein repair [5]. However, this technique uses the inguinal canal for entry to the preperitoneal space (PPS). Easy and long-term 
successful alternatives in inguinal hernia correction are needed because of the considerable proportions of chronic pain (15-40\%) after Lichtenstein's technique [6].

The TREPP technique was developed by Akkersdijk and is summarized in five principles:

1. Use a simple, easy-to-learn, and open technique, avoiding the scopic approaches with their considerable learning curves, severe adverse events, and lower cost effectiveness.

2. Stay away from the nerves and the inguinal canal during dissection.

3. Mesh positioning in the PPS, out of reach of the nerves.

4. No need for mesh fixation (because of the PPS mesh position).

5. No dissection nor reconstruction of the inguinal canal is necessary.

The aim of this report is to describe this new technique and its rationale by discussing the theoretical (dis-)advantages and the results of a pilot case series of 50 cases. This technique has already been performed in many patients in elective settings. The preperitoneal mesh technique in combination with the transrectus sheath approach has not been described before.

\section{Methods}

First, the patients were investigated at the outpatient department and an inguinal hernia was clinically assessed. Second, a standard preoperative screening by an anesthesiologist was undertaken.

\section{Surgical technique}

The TREPP technique can be performed under spinal anesthesia. To reach the PPS, a 5-cm straight incision is made about $1 \mathrm{~cm}$ above the pubic bone. The anterior rectus sheath is opened, as is the underlying fascia transversalis (Fig. 1). After retraction of the muscle fibers medially, the inferior epigastric vein and artery are identified and retracted medially as well. With a gentle movement of the dissecting finger, the PPS is created and a direct hernia can be immediately reduced. Using the iliac vessels as a landmark, the funiculus is identified with the spermatic cord, the testicular vessels, and a possible indirect hernia. The latter (if present) is now reduced. Using three long and thin retractors, a perfect PPS overview can be achieved and all possible hernia orifices (direct, indirect, and/or femoral) can be visualized. In the PPS, a self-expandable mesh is placed (Polysoft ${ }^{\circledR}$ 'Large', BARD Benelux, Belgium) that covers the complete myopectineum of Fruchaud. After deployment, the abdominal pressure keeps the mesh positioned without necessitating fixation. The anterior rectus sheath and the fascia of Scarpa are then closed with vicryl. The skin is closed intracutaneously with monocryl.

\section{Pilot study}

A prospective evaluation was carried out in 2006/2007 to assess the TREPP procedure's feasibility. Baseline characteristics and main outcome measures were evaluated directly and 2 years postoperatively. The authors were not yet aware of the European Hernia Society (EHS) Hernia Classification at the time of the operations in 2006/2007. A more descriptive classification was used at that time in the operation reports. All 50 patients were evaluated after at least 2 years postoperatively. Patients were interviewed by telephone concerning chronic pain complaints and/or limitations in daily life. Follow-up of at least 2 years was needed in order to confirm the theoretical benefits and feasibility.

\section{Results}

Fifty consecutive patients with a primary unilateral groin hernia underwent TREPP for inguinal hernia repair. The mean American Society of Anesthesiologists (ASA) classification was 1.2 (range 1-3). In a period of 5 months, 50 patients with primary unilateral groin hernias were operated. All patients were male, with a mean age of 54 years (range 24-81 years). The average skin-to-skin time was $20 \mathrm{~min}$, and the mean total theater time was $46 \mathrm{~min}$. Blood
Fig. 1 The intra-operative anterior view of the repaired groin hernia using the transrectus sheath preperitoneal (TREPP) technique
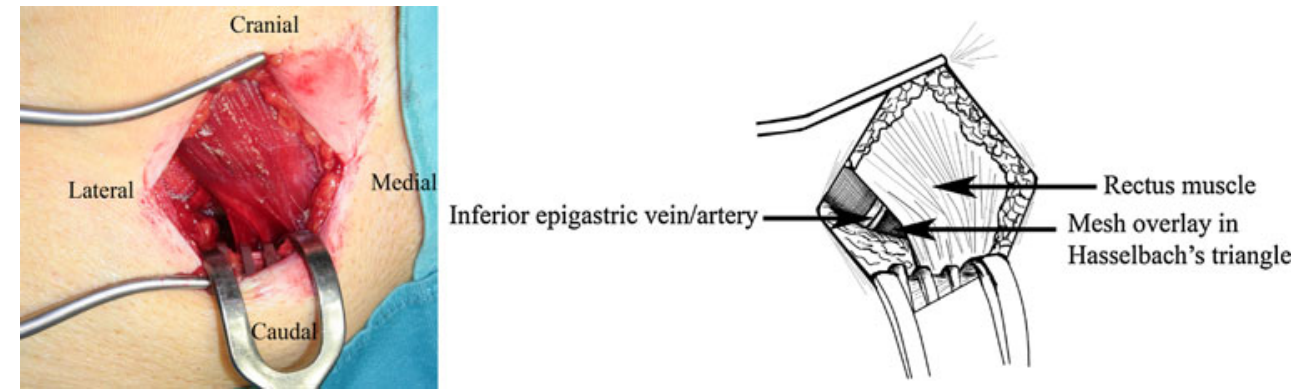
loss never exceeded $100 \mathrm{cc}$. There were 38 patients with a left-sided hernia $(76 \%), 49$ patients with a lateral hernia (98\%), and one patient with a scrotal hernia (2\%). Technical problems with TREPP did not occur. No conversions to other techniques (e.g., Lichtenstein, nor other open-mesh repairs) were necessary. Ninety-three percent of the patients were treated in the daycare setting. The mean postoperative pain did not exceed a visual analog scale (VAS) score of 4 (1-10 scale) in the first 14 days. Postoperative pain was controlled easily with paracetamol. Hematomas were observed in 18 patients (36\%), but never required secondary intervention. No wound infections occurred. No patients complained of any form of (chronic) pain nor the recurrence of symptoms 2 years postoperatively.

\section{Discussion}

The present $(n=50)$ pilot study shows that the TREPP technique is easy to learn in our experience and facilitates good primary outcome measures. Unfortunately, at the time of operation (2006/2007), the EHS Hernia Classification was not known yet among the authors and a more descriptive method of 'classification' was used. Presently, the standard operation form includes the EHS Hernia Classification, which is simple and easy to remember. Further studies are needed in order to confirm the outcomes from this TREPP pilot study. Future outcomes, together with the rationale behind this technique, may influence the future perspective on inguinal hernia repair. The rationale will be discussed according to five principle questions and their best available level of evidence [7] (LoE) in inguinal hernia repair.

Mesh rather than autologous inguinal hernia repair

During the last two decades, the use of mesh in inguinal hernia repair has become common practice since it was clearly demonstrated (LoE 1a) that, by using a mesh, the incidence of recurrences was diminished [8]. The open nonmesh techniques lost most of their popularity. Before the standard use of a mesh (e.g., Bassini's technique), recurrence was the most important outcome measure in inguinal surgery. A recent study shows recurrence rates of at least $8 \%$ after non-mesh repair using Bassini's technique [9].

The introduction of the mesh techniques such as Lichtenstein reduced recurrences ( $\mathrm{LoE} 1 \mathrm{a})$ [1]. The reinforcement of the inguinal canal can be positioned on top of the transversalis fascia (inlay), as is done in Lichtenstein's repair [6]. Despite reports about 'mesh shrinking' (or may that be 'wound contraction'?), the risk for recurrence after using the Lichtenstein technique is reported as being low (2\%) [10]. For placement of the mesh between the transver-
Table 1 Overview of the most often used mesh and non-mesh techniques for inguinal hernia repair

\begin{tabular}{lllll}
\hline Name & Mesh & Position & Approach & Technique \\
\hline McVay & No & - & Anterior & Open \\
Bassini & No & - & Anterior & Open \\
Shouldice & No & - & Anterior & Open \\
Lichtenstein & Yes & Inlay & Anterior & Open \\
Ugahary & Yes & Sublay & Posterior & Open \\
TIPP & Yes & Sublay & Anterior & Open \\
TREPP & Yes & Sublay & Posterior & Open \\
TEP & Yes & Sublay & Posterior & Endoscopic \\
TAPP & Yes & Sublay & Posterior & Laparoscopic \\
\hline
\end{tabular}

Sublay: in the preperitoneal space. Inlay: dorsal position in the inguinal canal. Mesh: prosthesis used in inguinal hernia repair

McVay: transition stitch incorporating the conjoined tendon, Cooper's ligament, the femoral sheath at the medial aspect of the femoral vein, and the inguinal ligament [16]

Bassini: the weakened inguinal floor is strengthened by approximating the conjoined tendon to the inguinal ligament from the pubic tubercle medially to the area of the internal ring laterally [16]

Shouldice: reconstruction in a four-layer overlap utilizing continuous fine-wire sutures. The defect is closed with multiple layers, none of which are placed with inordinate tension and completely obliterates the defect in the canal [16]

Lichtenstein: open/anterior approach tension-free mesh repair [17], global reference technique

Ugahary: a $4-\mathrm{cm}$ skin incision $3 \mathrm{~cm}$ craniolaterally to the internal inguinal ring through which a gridiron abdominal wall approach is used [16]

TIPP: open/anterior approach placing a mesh in the preperitoneal space through the annulus internus [3, 4]

TREPP: described in this article

TEP: endoscopic totally extraperitoneal placing of a mesh in the preperitoneal space [16]

TAPP: laparoscopic approach, through the abdominal cavity (transperitoneal/transabdominal) placing of a mesh in the preperitoneal space [16]

salis fascia and the peritoneum in the PPS ('upstream principle'), a laparoscopic (TAPP) or endoscopic (TEP) technique is most often used (Table 1).

Preperitoneal mesh position rather than onlay

Optimizing surgical techniques to improve outcomes and reduce the rate of recurrence is of great value to healthcare [11]. Biomechanically, the position of the mesh between the peritoneum and the abdominal wall muscles, the PPS should have advantages, especially when the mesh overlaps the abdominal wall defect widely. The intra-abdominal pressure causes the mesh to be pressed against the abdominal wall, keeping it positioned, rather than pushing it away. We call this the 'upstream principle'. In contrast, the inlay (or onlay) positioned mesh, as is done in the Lichtenstein 
technique, does not benefit from this physiological principle and needs fixation. Therefore, based on biomechanical principles, the preperitoneal placement may be preferable (LoE 2b).

Avoidance of inguinal nerve damaging caused by dissection or nerve entrapment

The risk of nerve damage through dissection of the inguinal canal, risk of nerve entrapment due to nerve suturing, or nerve fixation on the mesh is reduced to an absolute minimum in the TREPP technique. This is mainly because of the transrectus sheath approach, avoiding contact with the nerves and providing a total overview of the PPS (LoE 4). For example, the TIPP technique uses the inguinal canal for the entrance and creation of the PPS [3, 4]. The TIPP approach (by Pélissier) may be associated with less chronic pain and may have similar results concerning recurrence rates as the Lichtenstein technique [3-5].

Evolution of the TREPP technique involved combining several described techniques, such as Ugahary and the TIPP technique. It is important to realize that the rectus sheath has no posterior layer below the linea semilunaris-half way between the umbilicus and the pubic bone. Furthermore, in the most caudal part of the rectus abdominis muscle, the fibers run relatively parallel to the inguinal ligament. The chance of collateral damage to nerve tissue is, in our opinion, reduced to an absolute minimum secondary to avoiding the inguinal canal itself during dissection. The TREPP procedure may, therefore, theoretically reduce the risk for developing postoperative nerve-related chronic pain (LoE 5).

Open rather than endoscopic approach

Several techniques have been described to achieve the preperitoneal placement of a mesh in using an open approach [3-5]. Historically, most of the techniques carry the name of the surgeon who first described it (e.g., Stoppa). More recently, Kugel and Ugahary described techniques which involved splitting the oblique abdominal muscles in order to enter the PPS $[12,13]$. Recently, Pélissier described an open transinguinal preperitoneal hernia repair (TIPP). The TIPP technique uses the abdominal wall defect itself as the entrance point to the PPS, through which a preshaped selfexpandable hernia patch is introduced [3-5].

Since the endoscopic technique is possibly employed mostly for preperitoneal mesh placement, one could argue its superiority over the open techniques. Despite the published favorable results of both scopic approaches (TEP and TAPP), a number of reasons can be enumerated. In general, scopic procedures are considered to be technically demanding, may have long learning curves, and the use of the required disposable instruments may not be cost-effective (LoE 2b) [11, 14]. Furthermore, patients must be operated on under general anesthesia and, although complications are described as 'rare', visceral and major vascular injuries occur, as well as port-side hernias urging surgical correction. A considerable proportion of the TEP procedures result in adverse events $[11,15]$. These adverse events, which should be graded from the patient's perspective, have to be taken into account in decision-making and the development of new techniques have to be evaluated in studies with a low risk of bias [15].

Direct rather than indirect approach

The TREPP technique provides a complete overview of the PPS in our experience. Furthermore, the digital and tactile manipulation for creating the PPS has advantages compared to other techniques, such as Ugahary's. In our experience, the Ugahary technique leads to less preperitoneal visualization due to the indirect manipulation through speculae, retractors, and the lateral approach of the PPS (LoE 4).

Another TREPP advantage using the rectus sheath as the entrance point to the PPS is the direct vision of all possible hernia orifices, including the femoral hernia (LoE 4). The risk for an incisional hernia at this level is, theoretically, low because the entrance point is covered by a double layer consisting of muscle tissue and anterior rectus sheath. Moreover, the overlapping mesh may protect the abdominal wall from incisional hernia formation by covering the location of the rectus muscle in the PPS.

\section{Future perspectives and conclusions}

The evolution of all inguinal hernia repair techniques may conceptually lead to an open direct preperitoneal approach using a preperitoneal mesh position, such as TREPP. Despite the small number of patients, this pilot study shows that the TREPP technique may be a feasible method for hernia repair and seems to be promising. Based on the available evidence of systematic reviews supporting this evolution, and based on the favorable results of the first small series of TREPP patients, a randomized controlled trial is necessary to support the postulations derived from this pilot TREPP experience. Therefore, the TREPP technique will be evaluated in a randomized controlled trial in the near future.

\section{Conflict of interest None.}

Open Access This article is distributed under the terms of the Creative Commons Attribution Noncommercial License which permits any noncommercial use, distribution, and reproduction in any medium, provided the original author(s) and source are credited. 


\section{References}

1. Kumar S, Wilson RG, Nixon SJ, Macintyre IMC (2002) Chronic pain after laparoscopic and open mesh repair of groin hernia. $\mathrm{Br} \mathrm{J}$ Surg 89(11):1476-1479

2. Reinpold WM, Nehls J, Eggert A (2011) Nerve management and chronic pain after open inguinal hernia repair: a prospective two phase study. Ann Surg 254:163-168

3. Pélissier EP (2006) Inguinal hernia: preperitoneal placement of a memory-ring patch by anterior approach. Preliminary experience. Hernia 10:248-252

4. Pélissier EP, Blum D, Marre P, Damas JM (2001) Inguinal hernia: a patch covering only the myopectineal orifice is effective. Hernia 5:84-87

5. Berrevoet F, Maes L, Reyntjens K, Rogiers X, Troisi R, de Hemptinne B (2009) Transinguinal preperitoneal memory ring patch versus Lichtenstein repair for unilateral inguinal hernias. Langenbecks Arch Surg 395:557-562

6. Koning GG, de Schipper HJ, Oostvogel HJM, Verhofstad MHJ, Gerritsen PG, van Laarhoven KCJHM, Vriens PWHE (2009) The Tilburg double blind randomised controlled trial comparing inguinal hernia repair according to Lichtenstein and the transinguinal preperitoneal technique. Trials 10:89. doi:10.1186/17456215-10-89

7. Keus F, Wetterslev J, Gluud C, van Laarhoven KCJHM (2010) Evidence at a glance: error matrix approach for overviewing available evidence. BMC Med Res Methodol 10:90

8. McCormack K, Scott N, Go PM, Ross SJ, Grant A, Collaboration the EU Hernia Trialists (2003) Laparoscopic techniques versus open techniques for inguinal hernia repair. Cochrane Database
Syst Rev (1), Art. No.: CD001785. doi:10.1002/14651858. CD001785

9. Shi Y, Su Z, Li L, Liu H, Jing C (2010) Comparing the effects of Bassini versus tension-free hernioplasty: 3 years' follow-up. Front Med China 4(4):463-468

10. Koning GG, Koole D, de Jongh MAC, de Schipper JP, Verhofstad MHJ, Oostvogel HJM, Vriens PWHE (2011) The transinguinal preperitoneal hernia correction vs Lichtenstein's technique; is TIPP top? Hernia 15(1):19-22

11. Kuhry E, van Veen RN, Langeveld HR, Steyerberg EW, Jeekel J, Bonjer HJ (2007) Open or endoscopic total extraperitoneal inguinal hernia repair? A systematic review. Surg Endosc 21:161-166

12. Kugel RD (1999) Minimally invasive, nonlaparoscopic, preperitoneal, and sutureless, inguinal herniorrhaphy. Am J Surg 178(4):298-302

13. Ugahary F, Simmermacher RKJ (1998) Groin hernia repair via a grid-iron incision: an alternative technique for preperitoneal mesh insertion. Hernia 2:123-125

14. Wright D, O'Dwyer PJ (1998) The learning curve for laparoscopic hernia repair. In: Cuschieri A, MacFadyen BV Jr (eds) Seminars in laparoscopic surgery. WB Saunders, Philadelphia, PA, pp 227-232

15. Koning GG, Wetterslev J, van Laarhoven CJHM, Keus F (2011) PROTOCOL The totally extraperitoneal-(TEP) versus Lichtenstein's technique for inguinal hernia repair: a systematic review. Published online March 2011 at CTU website, http://www.ctu.dk

16. Matthews RD, Neumayer L (2008) Inguinal hernia in the $21 \mathrm{st}$ century: an evidence-based review. Curr Probl Surg 45:261-312

17. Amid PK, Shulman AG, Lichtenstein IL (1996) Open "tensionfree" repair of inguinal hernias: the Lichtenstein technique. Eur J Surg 162:447-453 